\title{
Soft processing for formation of self-assembled monolayer on hydrogen-terminated silicon surface based on visible-light excitation
}

\section{$\operatorname{AUTHOR}(\mathrm{S}):$}

Sano, Hikaru; Yaku, Tomohiro; Ichii, Takashi;

Murase, Kuniaki; Sugimura, Hiroyuki

\section{CITATION:}

Sano, Hikaru ...[et al]. Soft processing for formation of self-assembled monolaver on hydrogen-terminated silicon surface based on visible-light excitation. Journal of Vacuum Science \& Technology B: Microelectronics and Nanometer Structures 2009, 27(2): 858-862

\section{ISSUE DATE:}

2009-03

URL:

http://hdl.handle.net/2433/123515

\section{RIGHT:}

(c) 2009 American Vacuum Society; この論文は出版社版でありません。 引用の際には出版社版をご確認ご利用ください。; This is not the published version. Please cite only the published version. 
NM2-WeA5

\title{
Soft Processing for Formation of SAM on Hydrogen-Terminated Silicon Surface Based on Visible-Light Excitation
}

Hikaru Sano, Tomohiro Yaku, Takashi Ichii, Kuniaki Murase, and Hiroyuki Sugimura*

Department of Materials Science and Engineering, Kyoto University, Kyoto 606-8501, Japan

*E-mail address: hiroyuki-sugimura@mtl.kyoto-u.ac.jp

\section{MATERIALS}

1-hexadecene, silicon, fluoride ammonium

\begin{abstract}
Visible-light irradiation has been employed to promote a chemical reaction of 1-hexadecene with hydrogen-terminated silicon, yielding alkyl chains directly immobilized on bulk silicon surface. The resulting monolayer, that is, one type of self-assembled monolayer, was examined with water contact angle measurements, X-ray photoelectron spectroscopy, atomic force microscopy, and ellipsometry. While a part of the silicon surface was oxidized during the reaction, it was demonstrated that a compact monolayer was successfully formed both on $\mathrm{p}$ - and n-typed silicon substrates regardless of doping density.
\end{abstract}

\section{INTRODUCTION}


Organic thin films with a single molecular thickness are known to be formed via self-integration and self-organization of molecules chemisorbing onto solid surfaces. Studies of such self-assembled monolayers (SAMs) have attracted much attention due to fundamental scientific interest and their potential for practical applications. ${ }^{1)}$ One characteristic feature of the SAMs is that the orientation and arrangement of the molecules are highly ordered. Self-assembly has been recognized as a key for bottom-up nanotechnology to integrate a set of minute elements and to fabricate novel materials and devices. Organic functional materials are expected to play key roles in novel electric devices in the future, while current technologies are based on inorganics such as silicon semiconductors. Hence, a new technology to fuse and hybridize inorganic and organic materials is needed in the field of SAM formation.

From this point of view, SAMs of organic molecules on inorganic semiconductor surfaces $^{2-4)}$ are of great interest. SAMs can construct novel micro/nano electronic devices integrating a variety of functions based on organic molecules and semiconductor characteristics, considering the utility of silicon in semiconductor devices. It has been reported that SAMs can be formed on silicon substrates through silane coupling chemistry using a specific organosilane reagent as a precursor. ${ }^{5,6)}$ In this case the silicon substrate must be covered with an oxide layer, which is reactive to silane groups of precursor organosilane molecule. This oxide layer is, however, an insulating layer and thus prevents smooth electric linkage between the organic SAM and the silicon semiconductor.

In 1993, Linford et al. ${ }^{7)}$ reported a method to form SAMs on silicon substrates without an interfacial oxide layer. In this case, SAMs are directly immobilized on the silicon surface with covalent Si-C bonds, and the organic SAMs and the silicon semiconductor have an electric linkage. In the early research, Linford et al. used a radical initiator to form the 
SAMs: the radical initiator generates silicon radicals on the substrate surface, which react with the vinyl group of precursor alkene molecules and this reaction proceeds in a chain reaction to form SAMs on the surface. In 1995, the same group reported the formation of the same SAMs using heat treatment at $200{ }^{\circ} \mathrm{C}$ without a radical initiator. ${ }^{8)}$ After that, several processes to form similar SAMs have been developed ${ }^{9-12)}$ using, for example, alcohols $^{9)}$ and aldehydes ${ }^{10)}$ as precursor molecules and UV- and visible-light irradiation as activation methods.

After Linford et al. proposed the radical chain mechanism for SAM formation with radical initiator, the same mechanism was accepted for the heat treatment and UV-light irradiation methods without any obvious evidence, ${ }^{14)}$ although the radical chain reaction truly works as is evidenced by silicon radical formation using an STM-based excitation. ${ }^{15)}$ Actually, UV photons can dissociate the $\mathrm{Si}-\mathrm{H}$ bond ${ }^{16)}$ energetically. In the case of visible-light activation, however, the dissociation of $\mathrm{Si}-\mathrm{H}$ bond by visible-light is energetically impossible, and a plausible proposed mechanism is reaction between the vinyl groups and holes generated by photo irradiation. ${ }^{17-22)}$

In order to activate thermally the surface of hydrogen-terminated silicon (H-Si) for SAM formation, a temperature range of $100-200{ }^{\circ} \mathrm{C}$ is generally required. In this temperature range, however, some precursor molecules including biomolecules may be damaged. UV-light is more harmful for some kinds of precursor molecules, although the use of light has an advantage in that light can selectively illuminate a minute area on the substrate yielding SAMs grown on the selected area. ${ }^{10)}$ Therefore, the use of visible-light instead of heat treatment or UV-light irradiation is expected to considerably reduce such damage. Indeed, electrically-useful functional groups such as feroccenyl groups can be easily linked to the silicon surfaces with the visible-light activation method without damaging the precursor 
molecules. Detailed studies on the visible-light excitation of $\mathrm{H}-\mathrm{Si}$ substrates and the subsequent SAM formation are important, but information on this topic remains limited to a few reports. ${ }^{12,17-22)}$ We have already demonstrated that visible-light with any wavelength from $400 \mathrm{~nm}$ to $700 \mathrm{~nm}$ can activate the SAM formation. ${ }^{22)}$ This indicates that even molecules with an absorption band in the UV- or visible-light region can be directly attached onto silicon without any effects on the molecules by selecting an excitation wavelength range which would not be absorbed by the molecules.

Given that the SAMs are integrated into current silicon semiconductor electronics, the SAMs are to be formed on the various silicon substrate with different conduction types and impurity element densities. Studies focusing on the dopant effect on the SAM formation by light activation method have been conducted by Miramond et al. ${ }^{23)}$ and Sun et al., ${ }^{21)}$ but these reports did not prove the integrity of the resulting SAMs by using, for example, AFM and/or XPS. The objectives of the present study were to compare SAMs formed on H-Si(111) substrates with various dopant polarities and densities, to discuss the differences and similarities of these SAMs, and to elucidate the dopant dependence of the SAM formation.

\section{EXPERIMENTAL DETAILS}

\section{A Hydrogen-termination}

Both n- and p-typed silicon with various ranges of dopants were used in this study and the sample names and their polarity, dopant concentration, and relative resistivity are summarized in Table I. The dopant concentrations are determined from relative resistivity by using the graph ${ }^{24)}$ which shows the relationship between the two.

All substrates cut from the wafer were cleaned ultrasonically with ethanol and ultrapure water $(>18.0 \Omega \mathrm{cm}$ ) and then photochemically cleaned by exposure to vacuum ultraviolet 
light generated from an excimer lamp [Ushio, UER20-172 (UEM20-172) + UEP20, $\lambda=172$ $\mathrm{nm}, \mathrm{FWHM}=14 \mathrm{~nm}$, power density $\left.=10 \mathrm{~mW} \mathrm{~cm}^{-2}\right]$ for 20 min each. $^{25)} \mathrm{H}-\mathrm{Si}$ substrates were obtained by etching the cleaned samples in $5 \% \mathrm{HF}$ solution for 0 to $5 \mathrm{~min}$ at room temperature and subsequently in $40 \% \mathrm{NH}_{4} \mathrm{~F}$ solution for 30 to $40 \mathrm{~s}$ at $80{ }^{\circ} \mathrm{C}$ according to the polarity and dopant density of the substrate. ${ }^{26)}$ P-high samples easily suffer oxidation during the treatment such that many etch pits are generated on the surface, leaving a rough surface after treatment. To avoid this situation, in this study we added ammonium sulfite (ammonium sulfite monohydrate, Wako, 92\%) at a concentration of $0.025 \mathrm{M}$ in $40 \% \mathrm{NH}_{4} \mathrm{~F}$ solution and then the p-high sample was immersed in the $\mathrm{NH}_{4} \mathrm{~F}$ solution at $50{ }^{\circ} \mathrm{C}$ for 15 min. $^{27)}$ During the HF treatment, the experimental set-up was covered with aluminum foil serving as a light shield. $\mathrm{NH}_{4} \mathrm{~F}$ solution was heated to $50{ }^{\circ} \mathrm{C}$ or $80^{\circ} \mathrm{C}$ for oxygen removal. Throughout these treatments, the native oxide layer on each sample was removed and consequently the underlying silicon surface was exposed and terminated with hydrogen atoms.

\section{B SAM formation}

SAM formation, a hydrosilylation reaction between the vinyl group of 1-hexadecene (Tokyo Chemical Industry, 90\%, 99\%) and H-Si, was performed under visible-light activation. A custom-made quartz vessel was used consisting of a rectangular cell of 5-mm-thickness attached to one end of cylindrical tube with a diameter of about $20 \mathrm{~mm}$. Its capacity was about $100 \mathrm{~cm}^{3}$. Neat 1 -hexadecene liquid $\left(50 \mathrm{~cm}^{3}\right)$ was put into the vessel. Two glass tubes for purging with $\mathrm{N}_{2}$ gas were attached through a silicone rubber stopper set at the open end of the cylinder. The liquid was deaerated with a $\mathrm{N}_{2}$ gas stream for more than 30 min before immersion of $\mathrm{H}-\mathrm{Si}(111)$ substrate. After immersion for $30 \mathrm{~min}$, the substrate was 
irradiated with a xenon lamp [Asahi Spectra, MAX-1000 (UV-lamp + VIS-mirror)] from the outside of the cell through a long pass filter $(>420 \mathrm{~nm})$ for $8 \mathrm{~h}$. Light longer than $850 \mathrm{~nm}$ was cut off by the VIS-mirror. The liquid was kept at room temperature and deaerated throughout the experiment. The intensity of the visible-light was controlled at $265 \mathrm{~mW} \mathrm{~cm}^{-2}$ by using a neutral density filter installed inside the lamp housing. The power density was measured using a laser power meter (Neoark, PM335), on the light-receiving part of which cardboard with a $1 \times 1 \mathrm{~cm}^{2}$ square hole was attached. All the prepared samples were subsequently taken from the liquid and sonicated for 10 min each in hexane, methanol, and ultrapure water, in that order.

\section{Analytical methods}

The static water contact angles of the resulting samples were measured with a contact angle meter (Kyowa Interface Science, Model CA-D). The size of the water droplets was fixed at about $1.5 \mathrm{~mm}$ in diameter. X-ray photoelectron spectroscopy (XPS) analysis was carried out using an ESCA-3400 system (Kratos Analyical), the background pressure of which was less than $5 \times 10^{-6} \mathrm{~Pa}$. The $\mathrm{Mg} \mathrm{K \alpha}$ X-ray source was operated at $10 \mathrm{kV}$ and $10 \mathrm{~mA}$. Quantitative analysis was carried out by using only the $\mathrm{Si}(2 \mathrm{p}), \mathrm{C}(1 \mathrm{~s})$, and $\mathrm{O}(1 \mathrm{~s})$ regions on all samples. The XPS spectra obtained were calibrated so that $\mathrm{Si}-\mathrm{Si}$ peaks from the $\mathrm{Si}$ substrates were referenced to $99.6 \mathrm{eV}^{28)}$ in order to cancel binding energy shifts due to charging up effects. To make the chemical state conspicuous, the intensity scales of the $\mathrm{Si}(2 \mathrm{p})$ spectra were standardized so that the main $\mathrm{Si}-\mathrm{Si}$ peaks became the same intensity. Topographic images with an area of $500 \times 500 \mathrm{~nm}^{2}$ were acquired by an atomic force microscope (AFM; Seiko Instruments, SPA-300HV + SPI-3800N) with a silicon probe (Seiko Instruments, SN-AF01-100, force constant of $0.1 \mathrm{~N} \mathrm{~m}^{-1}$ ). The thicknesses of the resultiing 
SAMs were measured with a spectroscopic ellipsometer (Otsuka Electronics, FE-5000). The region measured was $400-800 \mathrm{~nm}$ in wavelength and the incident angle was set at $70^{\circ}$. The model of air / organic film / silicon was used for the analysis of raw data. The refractive index of $\mathrm{SiO}_{2}{ }^{29)}$ was adopted as that of the organic film over the measured wavelength range.

\section{RESULTS AND DISCUSSION}

\section{A Hydrogen-termination}

All obtained surfaces showed a water contact angle of around $82^{\circ}$, indicating that the surface silicon atoms were terminated with hydrogen atoms and in good agreement with the reported value. ${ }^{30)}$ Table II shows the amounts of each element obtained from XPS quantitative analysis of the hydrogen-terminated samples with different dopant polarities and dopant densities, and they show only around 5 at.\% of oxygen atoms, showing that surface oxide layer was successfully removed through hydrogen-termination treatment. Several percentages of oxygen atoms and carbon atoms were detected on the surface due to the absorbed water and contamination respectively. XPS Si(2p) spectra had only one peak, which comes from bulk silicon, also meaning that native oxide layer was completely removed by hydrogen-termination treatment and implies that the surface was terminated with hydrogen atoms. Fig. 1a displays the spectrum of an n-high sample after hydrogen-termination process as a representative of the other samples. There is a peak at $99.6 \mathrm{eV}$ of binding energy, which comes from bulk silicon. The binding energy around $103.8 \mathrm{eV}$ is the region for silicon oxide, and no meaningful peaks are seen there. All of the AFM topographic images shown in Fig. 2 display stair-like structures of terraces and steps, but the image of the p-high sample looks different from the others. This is due to the difficulty in $\mathrm{NH}_{4} \mathrm{~F}$ etching for the case of the p-high sample because the sample surface easily suffers oxidation by 
dissolved oxygen. To avoid the oxidation, traces of sulfite ion were added in $\mathrm{NH}_{4} \mathrm{~F}$ solution (see experimental section). The step height is measured to be around $0.3 \mathrm{~nm}$ which is in good agreement with a monoatomic step on Si (111) plane on all samples. Terrace widths are all around $60 \mathrm{~nm}$, which is in good agreement with the mis-cut angle of the wafers. Here it should be emphasized that all samples with different dopant polarities and dopant densities are successfully hydrogen-terminated.

\section{B SAM formation}

The samples after SAM formation treatment with 1-hexadecene show hydrophobicity irrespective of the property of the substrate by water contact angle measurement. Some values are not close to the value of closely-packed methyl terminated surfaces, $110^{\circ}{ }^{31)}$ but more than that of their initial hydrogen-terminated state shown in Table III.

When focusing on the results of XPS quantitative analysis, the carbon amounts at the surfaces increased greatly up to 25 - 33 at.\%, compared with that of the H-Si(111) sample, around 5 at.\%. Accordingly, 1-hexadecene molecules were successfully attached to the H-Si(111) substrate surfaces resulting in the formation of hexadecyl SAMs. Furthermore, visible-light could excite the SAM formation reaction on all the hydrogen-terminated surfaces with different dopant polarities and dopant densities. Here we should note that surface oxidation also occurred during SAM formation treatment in all samples, as shown by the slight increase in oxygen content. Fig $1 \mathrm{~b}$ is the $\operatorname{Si}(2 \mathrm{p})$ spectrum of an n-high sample after SAM formation treatment as a representative of the spectrum of all other silicon samples. The spectrum has two peaks. One is at a binding energy of $99.6 \mathrm{eV}$ and this component comes from bulk silicon. The other small and broad peak around binding energy of 103.8 
$\mathrm{eV}$ comes from silicon oxide: This component is small indicating that the surface reacted mostly with precursor molecules during SAM formation treatment and was rarely oxidized.

The thicknesses of the SAMs were estimated to be $1.9-2.4 \mathrm{~nm}$ by ellipsometry. These values are reasonable for the thickness of monolayers, since the precursor molecule of 1-hexadecene has a length of about $2 \mathrm{~nm}$. The thickness of a monolayer is related to the tilt angle of the adsorbed molecules and in this case the organic molecules comprising these SAMs are arranged with a high order on each substrate irrespective of different dopant concentrations.

AFM topographic images of the samples after SAM formation treatment are shown in Fig. 3. They are very similar to that of the H-Si(111) surface (Fig. 2). Monoatomic steps of the H-Si(111) substrates remain without any distortions even though the substrates were coated with a SAM, the thickness of which was more than 7 times greater than the step height. This result demonstrates that the prepared SAMs were all uniform and highly ordered enough.

In contrast, Miramond et al. ${ }^{23)}$ reported on SAM formation using UV-light, where SAM is formed on all silicon samples with various dopant densities but SAM is not formed on n-typed silicon with relative resistivity of $0.005 \Omega \mathrm{cm}$, which corresponds to the n-high sample in our case. On the n-high sample, SAM was formed by visible-light irradiation in our study, as elucidated by contact angle measurements, XPS, and AFM.

Let us now look at the dopant density effect on SAM formation in detail. Comparing SAMs on n-typed sample with SAMs on p-typed sample, SAMs on n-type sample were rather well-ordered and densely-packed. SAM on p-high silicon surface was not so well ordered and densely packed, in particular. The fact that the dopant polarity affect the molecular density and ordering of the SAM is accounted for by thinking the band-bending on silicon surface $^{12)}$ : Light irradiation on the silicon surface create electrons and holes in the bulk, 
which are separated by the electric field that are typically found in the subsurface space charge region. The hydrogen-terminated n-typed silicon samples exhibit upward band-bending near the surface, such that photoexited electrons drift into the bulk and photoexited holes drift towards the surface, where they can induce reactions of a vinyl group with the H-terminated surface. Conversely, p-typed samples typically exhibit downward band-bending, such that photoexited holes drift away from the surface. When a vinyl group reacts with the hydrogen-terminated surface, vinyl groups are nucleophilic so that the vinyl group tend to react on the surface with more positive-charged surface ${ }^{22)}$. This is the reason why n-typed silicon surface is susceptible to SAM formation. But this can not account for the result that SAM on n-high silicon surface is less ordered and less densely packed than SAM on n-low silicon surface, since for n-typed samples the driving force for the holes to migrate to the surface would increase with the dopant density so that $n$-high sample would have the highest SAM formation efficiency. This is plausibly because of the difference in the surface roughness. The low molecular density of SAM on p-high silicon surface is also plausibly because of the difference in the surface roughness.

\section{CONCLUSION}

We have examined the possibility of SAM formation from 1-alkene molecules on various kinds of hydrogen-terminated silicon substrate. In particular, we focused on the order of the arrangement of the absorbed molecules by using AFM. From the results described above, we conclude that visible-light certainly promoted the SAM formation between hydrogen-terminated silicon and 1-alkene, irrespective of the dopant polarity and density of the silicon sample. The resulting SAMs are proven to have almost the same features as each other. Comparing the SAMs on n-typed silicon with p-typed silicon, the 
SAMs n-typed silicon are rather well ordered and densely packed, which is accounted for by taking band-bending ${ }^{12)}$ and surface roughness into consideration. Visible-light certainly promotes SAM formation on n-typed highly doped silicon, while UV-light could not ${ }^{23)}$. Thus, the visible-light activation method has some advantages over the other methods.

\section{ACKNOWLEDGMENTS}

We thank Dr. K. Kobayashi and Professor S. Kurokawa at Kyoto University for their help in preparing hydrogen-terminated silicon substrates. This work was partially supported by a Grant-in-Aid for the Global COE Program, "International Center for Integrated Research and Advanced Education in Materials Science", Grant-in-Aid for Scientific Research (KAKENHI) No. 19049010 on Priority Areas, "Strong Photons-Molecules Coupling Fields (470)," and KAKENHI (B) No. 20360314, from the Ministry of Education, Culture, Sports, Science, and Technology of Japan. 


\section{REFERENCES}

1) A. Ulman, Chem. Rev. 96, 1533 (1996).

2) H. Sugimura, in Nanoclistalline Materials Their Synthesis-Structure-Property Relationships and Applications, ed. S. C. Tjong (Elsevier, Oxford, 2006) Chap. 3.

3) P. R. Moses and R. W. Murray, J. Am. Chem. Soc. 98, 7435 (1976).

4) T. Osa and M. Fujihira, Nature 264, 349 (1976).

5) J. Sagiv, J. Am. Chem. Soc. 102, 92 (1980).

6) S. R. Wasserman, Y.-T. Tao, and G. M. Whitesides, Langmuir 5, 1074 (1989).

7) M. R. Linford and C. E. D. Chidsey, J. Am. Chem. Soc. 115, 12631 (1993).

8) M. R. Linford, P. Fenter, P. M. Eisenberger, and C. E. D. Chidsey, J. Am. Chem. Soc. 117,3145 (1995).

9) R. Boukherroub, S. Morin, P. Sharpe, and D. D. M. Wayner, Langmuir 16, 7429 (2000).

10) F. Effenberger, G. Götz, B. Bidlingmaier, and M. Wezstein, Angew. Chem., Int. Ed. 37, 2462 (1998).

11) J. M. Buriak, Chem. Rev. 102, 1271 (2002).

12) W. Cai, Z. Lin, T. Strother, L. M. Smith and R. J. Hamers, J. Phys. Chem. B, 106, $2656(2002)$.

13) M. R. Kosuri, H. Gerung, Q. Li, S. M. Han, B. C. Bunker, and T. M. Mayer, Langmuir 19, 9315 (2003).

14) C. Coletti, A. Marrone, G. Giorgi, A. Sgamellotti, G. Cerofolini, and N. Re, Langmuir 22, 9949 (2006).

15) G. P. Lopinski, D. D. M. Wayner, and R. A. Wolkow, Nature 406, 48 (2000). 
16) S. Pai and D. Doren, J. Phys. Chem. 98, 4422 (1994).

17) M. P. Stewart and J. M. Buriak, J. Am. Chem. Soc. 123, 7821 (2001).

18) Q.-Y. Sun, L. C. P. M. de Smet, B. van Lagen, A. Wright, H. Zuilhof, and E. J. A. R. Sudhölter, Angew. Chem., Int. Ed. 43, 1352 (2004).

19) B. J. Eves, Q.-Y. Sun, G. P. Lopinski, and H. Zuilhof, J. Am. Chem. Soc. 126, $14318(2004)$.

20) L. C. P. M. de Smet, G. A. Stork, G. H. F. Hurenkamp, Q.-Y. Sun, H. Topal, J. E. Vronen, A. B. Sieval, A. Wright, G. M. Visser, H. Zuilhof, and E. J. R. Sudhölter, J. Am. Chem. Soc. 125, 13916 (2003).

21) Q.-Y. Sun, L. C. P. M. de Smet, B. van Lagen, M. Giesbers, P. C. Thüne, J. van Engelenburg, F. A. de Wolf, H. Zuilhof, and E. J. R. Sudhölter, J. Am. Chem. Soc. 127, 2514 (2005).

22) H. Sano, H. Maeda, S. Matsuoka, K. -H. Lee, K. Murase and H. Sugimura, Jpn. J. Appl. Phys. 47, 5659 (2008).

23) C. Miramond and D. Vuillaume, J. Appl. Phys. 96, 1529 (2004).

24) S. M. Sze, Semiconductor Devices -Physics and Technology- (Wiley, New York, 1981) 2nd ed., p.49 [in Japanese].

25) H. Sugimura, A. Hozumi, T. Kameyama, and O. Takai, Surf. Interface Anal. 34, $550(2002)$

26) S. Kurokawa, T. Takei, and A. Sakai, Jpn. J. Appl. Phys. 42, 4655 (2003).

27) H. Fukidome and M. Matsumura, Appl. Surf. Sci. 130 - 132, 146 - 150 (1998).

28) A. Lehner, G. Steinhoff, M. S. Brandt, M. Eickhoff, and M. Stutzmann, J. Appl. Phys. 94, 2289 (2003). 
CA, 1998).

30) Y. Sato and M. Maeda, Jpn. J. Appl. Phys. 33, 6508 (1994).

31) A. Ulman, An Introduction to Ultrathin Organic Films from Langmuir-Blogett to Self-Assembly (Academic Press, San Diego, CA, 1991) p. 281. 
Fig 1

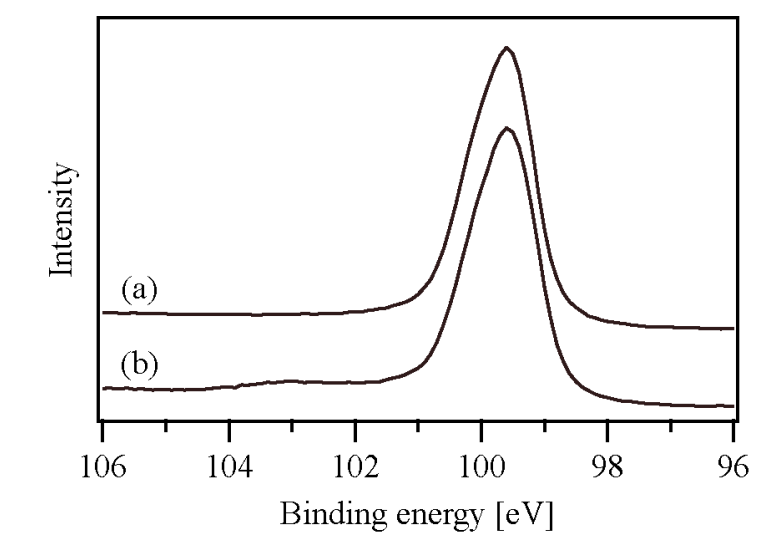


Fig. 2
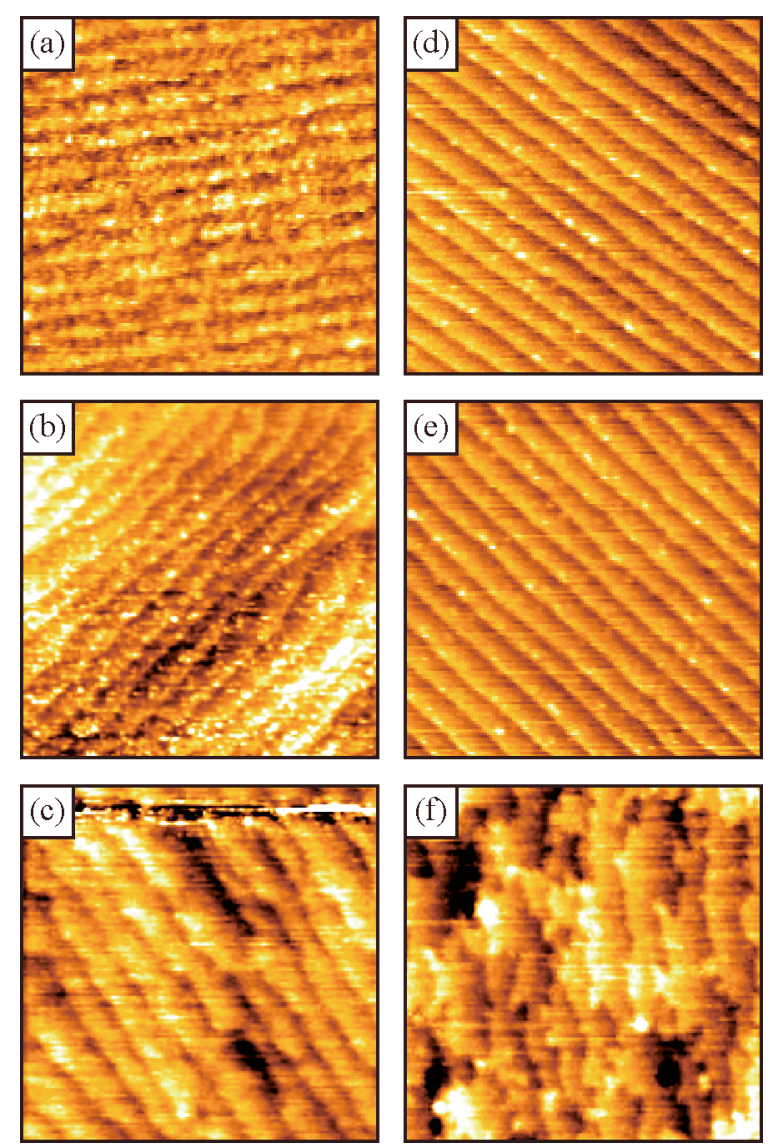
Fig. 3
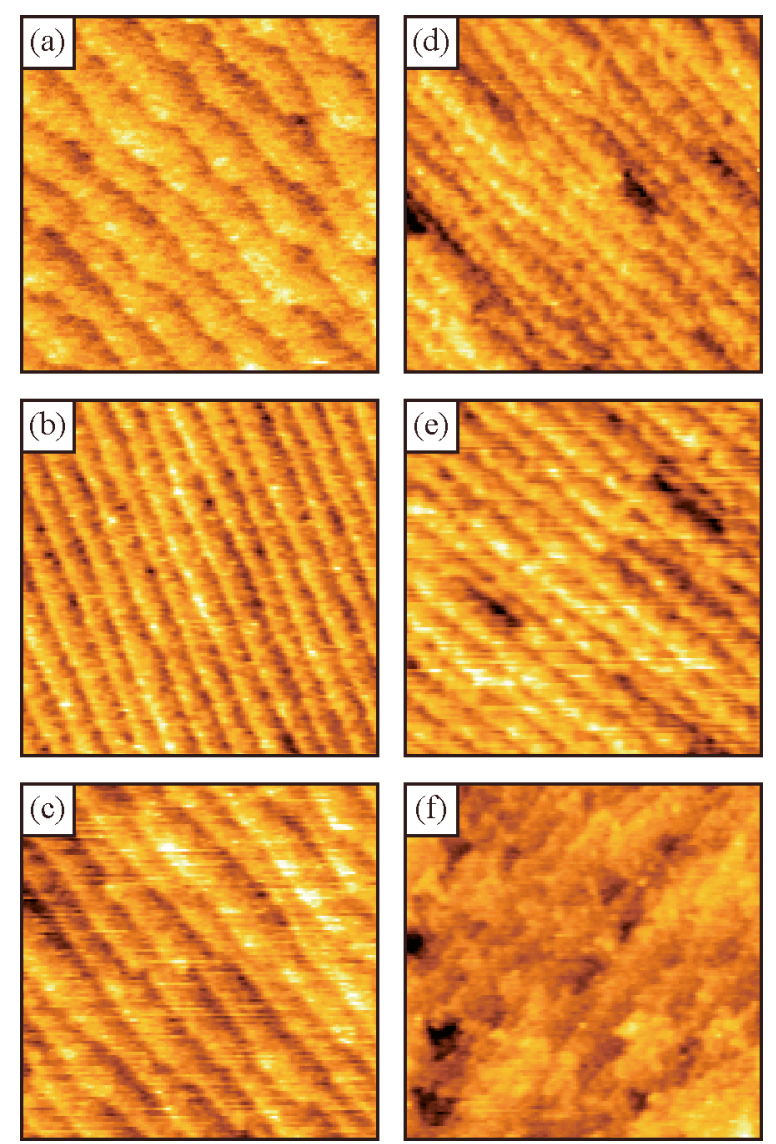
Table I

\begin{tabular}{lcccc} 
Sample name & Polarity & $\begin{array}{c}\text { Impurity } \\
\text { element }\end{array}$ & Dopant density $\left[\mathrm{cm}^{-3}\right]$ & $\begin{array}{c}\text { Relative resistivity } \\
{[\mathrm{ohm} \mathrm{cm}]}\end{array}$ \\
\hline n-low & $\mathrm{n}$ & Phos. & $6 \times 10^{14}$ & $6.44-7.46$ \\
\hline n-mid & $\mathrm{n}$ & Phos. & $8 \times 10^{15}$ & $0.15-0.56$ \\
\hline n-high & $\mathrm{n}$ & As & $7 \times 10^{19}-1 \times 10^{20}$ & $0.001-0.004$ \\
\hline p-low & $\mathrm{p}$ & Boron & $4 \times 10^{14}$ & $16.5-17.6$ \\
\hline p-mid & $\mathrm{p}$ & Boron & $1 \times 10^{17}$ & $0.140-0.145$ \\
\hline p-high & $\mathrm{p}$ & Boron & $1 \times 10^{20}-2 \times 10^{20}$ & $0.001-0.004$ \\
\hline
\end{tabular}


Table II

\begin{tabular}{cccc} 
& XPS [at.\%] & & \\
\cline { 2 - 4 } & Si 2p & C 1s & O 1s \\
\hline n-low & 92.9 & 5.3 & 1.8 \\
\hline n-mid & 93.3 & 4.9 & 1.8 \\
\hline n-high & 94.3 & 3.7 & 2.0 \\
\hline p-low & 98.3 & 1.1 & 0.6 \\
\hline p-mid & 86.0 & 6.3 & 7.8 \\
\hline p-high & 92.1 & 5.3 & 2.6
\end{tabular}


Table III

\begin{tabular}{lccccc} 
& \multicolumn{2}{c}{ XPS $[$ at.\%] } & & \\
\cline { 3 - 6 } & C.A. $\left[{ }^{\circ}\right]$ & Si 2p & C 1s & O 1s & thickness $[\mathrm{nm}]$ \\
\hline n-low & 107.5 & 59.2 & 33.4 & 7.4 & 2.4 \\
\hline n-mid & 100.2 & 67.6 & 24.8 & 7.6 & 1.9 \\
\hline n-high & 102.1 & 62.3 & 25.3 & 12.4 & 2.2 \\
\hline p-low & 99.2 & 63.3 & 27.7 & 8.9 & 2.2 \\
\hline p-mid & 100.0 & 60.5 & 30.0 & 9.6 & 2.2 \\
\hline p-high & 95.0 & 64.3 & 26.4 & 9.3 & 2.2
\end{tabular}




\section{CAPTIONS}

Fig. 1

XPS Si(2p) spectra of $n$-high sample (a) before and (b) after SAM formation treatment.

Fig. 2

AFM topographic images of (a) n-low, (b) n-mid, (c) n-high (d) p-low, (e) p-mid, and (f) p-high samples after hydrogen-termination treatment. All samples have stair-like surface structures. Image sizes are all $500 \mathrm{~nm} \times 500 \mathrm{~nm}^{2}$.

Fig. 3

AFM topographic images of (a) n-low, (b) n-mid, (c) n-high (d) p-low, (e) p-mid, and (f) p-high samples after SAM formation treatment. All samples have stair-like surface structures, similar to that of samples after hydrogen-termination treatment, meaning the absorbed molecules have a highly ordered arrangement. Image sizes are all $500 \mathrm{~nm} \times 500$ $\mathrm{nm}^{2}$

Table I

Sample names and their polarity, dopant concentration, and relative resistivity. The dopant concentration are determined from relative resistivity by using the relationship between the two in ref. 24.

Table II

The quantitative value of the elements from XPS analysis of (a) n-low, (b) n-mid, (c) n-high (d) p-low, (e) p-mid, and (f) p-high samples after hydrogen-termination treatment.

Table III

The quantitative value of the elements from XPS analysis of (a) n-low, (b) n-mid, (c) n-high (d) p-low, (e) p-mid, and (f) p-high samples after SAM formation treatment. 